\title{
Dynamics of the major disruption of a DIII-D plasma ${ }^{a)}$
}

\author{
S. E. Kruger ${ }^{\text {b) }}$ \\ Tech-X Corporation, 5621 Arapahoe Avenue, Suite A, Boulder, Colorado \\ D. D. Schnack \\ SAIC, 10210 Campus Point Drive, San Diego, California \\ C. R. Sovinec \\ University of Wisconsin-Madison, 1500 Engineering Drive, Madison, Wisconsin
}

(Received 29 November 2004; accepted 21 January 2005; published online 20 April 2005)

\begin{abstract}
The dynamics of the major disruption of DIII-D discharge 87009 are investigated with the NIMROD code [Sovinec et al., J. Comput. Phys. 195, 355 (2004)]. To explore the time dynamics in a computationally efficient manner, a fixed-boundary equilibrium is used to model the physics of a plasma being heated through an ideal magnetohydrodynamic (MHD) instability threshold. This simulation shows a faster-than-exponential increase in magnetic energy as predicted by analytic theory [Callen et al., Phys. Plasmas 6, 2963 (1999)]. The dynamics of the heat flux loading on the divertor surfaces is explored with an equilibrium that has the plasma beta raised $8.7 \%$ above the best equilibrium reconstruction to start above the ideal MHD threshold. The nonlinear evolution of the ideal mode leads to a stochastic magnetic field and parallel heat transport leads to a localization of the heat flux that is deposited on the wall. The structure of the heat flux deposition is dependent upon the magnetic topology that results from the growth of the ideal mode. (C) 2005 American Institute of Physics. [DOI: 10.1063/1.1873872]
\end{abstract}

\section{INTRODUCTION}

During tokamak experimental operation, events that rapidly terminate the plasma discharge occasionally occur. The complete and rapid loss of thermal and magnetic energy in these disruptions results in large thermal and magnetic loads on the material wall. For proposed next step experiments such as the International Thermonuclear Experimental Reactor, the stored energy will be approximately 100 times greater than present day devices ${ }^{1}$ greatly increasing the potential damage of these events. Exacerbating the risk to the machine and increasing the engineering challenges, the disruption phenomena are often highly non-axisymmetric; ${ }^{2}$ this results in localized deposition of the heat loads. Understanding the onset mechanisms and the nonlinear dynamics leading to the disruption is crucial for understanding ways to prevent or mitigate disruptions.

Disruptions are generally placed in one of two categories depending on the sequence of events that occur. ${ }^{2}$ In major disruptions, the thermal energy is lost first in the thermal quench phase. The current density profile flattens, and the resultant change in the internal inductance causes the total plasma current to increase. A current quench from the cold plasma then occurs. These types of disruptions are generally caused by long wavelength, non-axisymmetric magnetohydrodynamic (MHD) instabilities. Because the thermal quench generally occurs first, the heat flux normal to the material wall surface is of primary concern for understanding the implication of major disruptions in burning plasma experiments; in particular, greater understanding of the local-

\footnotetext{
a) Paper RI1 4, Bull. Am. Phys. Soc. 49, 325 (2004).

${ }^{b)}$ Invited speaker. Electronic mail: kruger@txcorp.com
}

ization of heat flux as observed in divertor temperature measurements, and of how the point of maximum heat flux moves away from the original strike points is needed. In the other type of disruption, the vertical displacement event (VDE), the plasma moves vertically to strike the material wall. A thermal quench occurs followed by a current quench without an increase in the current. In general, simulations of major disruptions are more difficult because the initial motion of the VDE is axisymmetric, reducing the toroidal resolution required.

The experimental phenomenology of the major disruption in DIII-D discharge 87009 has attracted a great deal of experimental and theoretical interest. ${ }^{3-8} \mathrm{~A}$ combination of analytic theory ${ }^{3}$ and linear ideal MHD code analysis ${ }^{4}$ has been successful in predicting both the time scale of the disruption $^{3}$ and the spatial structure ${ }^{4}$ of the mode. The success of the model and the indication that the phenomenology can be described strictly with a magnetohydrodynamic model makes this an attractive case to study with the NIMROD $^{9}$ nonlinear initial-value code. Unlike the simple analytic/linear numerical MHD model, an initial-value code allows for detailed studies of the mechanism leading to the loss of plasma confinement and the resultant heat deposition on the plasma wall.

In this paper, two different aspects of the dynamics of the major disruption in DIII-D discharge 87009 are explored using the NIMROD code. Before discussing the simulations, a detailed discussion of the model including its strengths and limitations is presented. Then the first set of simulations, which investigate the time dynamics using fixed-boundary equilibria, is presented. In the next section, the NIMROD simulation starts from a free-boundary equilibrium that is 
above the ideal MHD threshold. The emphasis in this simulation is to model how the heat flux gets deposited on the wall. In the final section, conclusions are drawn and further work is discussed.

\section{SIMULATION MODEL}

The fluid equations used in these studies are evolution equations for density, $n$, flow, $\mathbf{V}$, current, $\mathbf{J}$, and total ion and electron temperatures, $T_{i}$ and $T_{e}$ :

$$
\begin{aligned}
& \frac{\partial n}{\partial t}+\boldsymbol{\nabla} \cdot n \mathbf{V}=0 \\
& m_{i} n\left(\frac{\partial \mathbf{V}}{\partial t}+\mathbf{V} \cdot \boldsymbol{\nabla} \mathbf{V}\right)=-\boldsymbol{\nabla} p+\mathbf{J} \times \mathbf{B}+\boldsymbol{\nabla} \cdot \mu m_{i} n \boldsymbol{\nabla} \mathbf{V}
\end{aligned}
$$

$$
\begin{aligned}
\frac{n}{\gamma-1}\left(\frac{\partial T_{\alpha}}{\partial t} \mathbf{V}_{\alpha} \cdot \boldsymbol{\nabla} T_{\alpha}\right)= & n T_{\alpha} \boldsymbol{\nabla} \cdot \mathbf{V}_{\alpha}+\boldsymbol{\nabla} \cdot n\left[\left(\kappa_{\|}-\kappa_{\perp}\right) \hat{\mathbf{b}} \hat{\mathbf{b}}\right. \\
& \left.+\kappa_{\perp} I\right] \cdot \boldsymbol{\nabla} T_{\alpha}-Q_{\alpha},
\end{aligned}
$$

$$
\mathbf{E}+\mathbf{V} \times \mathbf{B}=\eta J,
$$

$$
\mu_{0} \mathbf{J}=\nabla \times \mathbf{B},
$$

where $\mathbf{E}$ and $\mathbf{B}$ are the electric, kinetic, and magnetic fields, respectively; and $\eta, \mu$, and $\kappa$ is the electric, kinetic, and thermal diffusivities, respectively; and $Q$ is a heat source. In Eq. (3), the Ohmic heating and stress-tensor heating have been neglected because our simulation times are much less than the transport time scales. These equations, in conjunction with Maxwell's equations, are closed because the closures have been specified for the stress tensors, $\Pi_{\alpha}$, and the conductive heat flows, $\mathbf{q}_{\alpha}$. Specifically, $\Pi_{i}=n m_{i} \mu \boldsymbol{\nabla V}$ and $\Pi_{e}$ $=0$ have been used for the ion and electron stress tensors, and a simplified model of the Braginskii heat flux has been used for the heat flux closure:

$$
q=-n \kappa_{\|} \hat{\mathbf{b}} \hat{\mathbf{b}} \cdot \nabla T-\kappa_{\perp} \boldsymbol{\nabla}_{\perp} T .
$$

The rapid equilibration of temperature along field lines is modeled by having $\kappa_{\|} \gg \kappa_{\perp}$.

The resistive MHD equations neglect the whistler, Hall, and electron inertia terms, i.e., the two-fluid physics in the generalized Ohms law, Eq. (4). As discussed in Sec. IV, the thermal quench of the plasma disruption is caused by a rapid stochasticization of the magnetic field. The change in the topology of the magnetic field is a result of magnetic reconnection that is known to be sensitive to the model used in the generalized Ohms law ${ }^{10}$ with two-fluid physics generally giving faster reconnection than resistive MHD. Resolving the length scales necessary in a two-fluid model over the entire plasma region as the magnetic field becomes stochastic presents a large numerical challenge. The successful application of linear MHD codes to analyze the experimental results ${ }^{4}$ gives confidence that intuitive insight into the dynamics can be gained with the nonlinear, resistive MHD model.
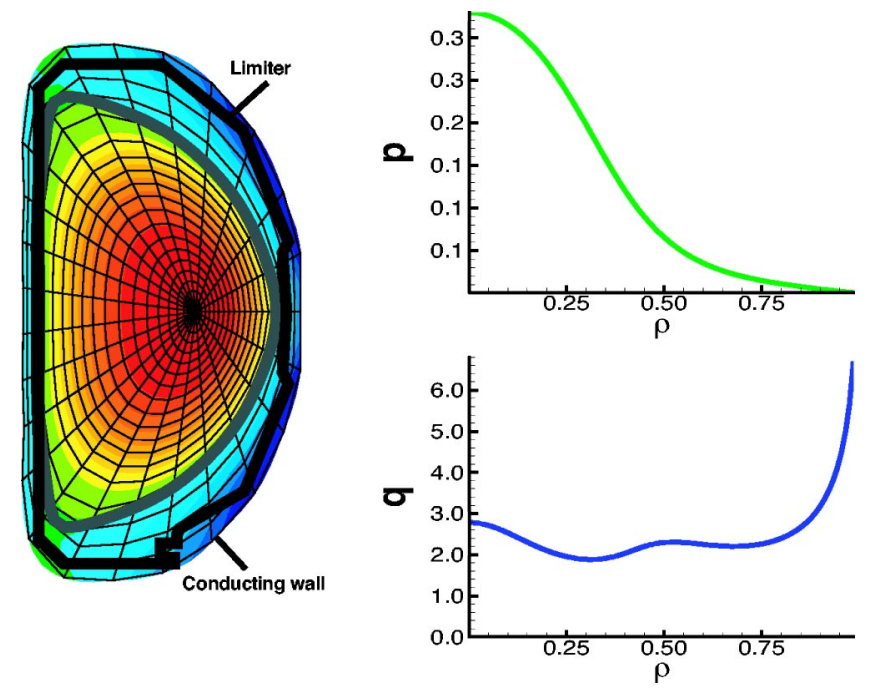

FIG. 1. (Color online). Equilibrium reconstruction of DIII-D discharge 87009 at $1675 \mathrm{~ms}$ shows a reverse shear profile and peaked pressure profile in a highly triangulated plasma. The plasma region contains a hot region where the field lines are closed and a cold, halo plasma where the field lines strike the wall.

To minimize the interactions of the hot plasma with the cold wall, modern tokamaks divide the plasma into two distinct regions, as seen in Fig. 1: a core region where field lines close upon themselves and confine the plasma $\left(T_{e} \sim 10 \mathrm{keV}\right.$, $n \sim 10^{19} \mathrm{~m}^{-3}$ in DIII-D), and a halo region where the field lines intersect the wall and the plasma remains cold $\left(T_{e}\right.$ $\sim 1-10 \mathrm{eV}, \dot{n} \sim 10^{16} \mathrm{~m}^{-3}$ in DIII-D). Using a Spitzer resistivity $\left(\eta \sim T^{-3 / 2}\right)$ in the halo region gives large values that suppress currents. The separatrix that divides the two regions is only clearly defined with two-dimensional magnetic fields; with three-dimensional magnetic fields a stochastic region generally exists that blurs the separation and increases the modeling difficulty. To avoid the numerical difficulties associated with the large three-dimensional gradients, the majority of magnetohydrodynamic simulations in tokamaks have placed the conducting wall on the last-closed flux surface. These fixed-boundary simulations have the added advantage that inverse Grad-Shafranov solvers ${ }^{11}$ can be used to give highly accurate, easily specified equilibria. A prominent example of a fixed-boundary major disruption simulation showing a thermal quench is the kink-ballooning simulation of Park. ${ }^{12}$

Free-boundary simulations, which have the computational boundary beyond the last-closed flux surface, have primarily been used for VDE disruptions ${ }^{13,14}$ or studies of liquid walls, ${ }^{15}$ where the dominant axisymmetric nature of the instability gives weak stochasticity (or none if only the $n=0$ component is kept). For more non-axisymmetric modes, accurate modeling of rapid parallel equilibration in three dimensions is required to resolve the distinction between the halo region and core region as the plasma evolves nonlinearly and becomes highly stochastic. The use of high-order finite elements in NIMROD ${ }^{9}$ has allowed numerical simulations of ratios of $\kappa_{\|} / \kappa_{\perp}$ never before possible, and the simulations presented here use values of $\kappa_{\|} / \kappa_{\perp}=10^{8}$. This new capability allows accurate free-boundary simulations of ma- 
jor disruptions to be computationally feasible. In the simulations below, fixed-boundary simulations are used to explore the time dynamics because accurate control of the equilibrium is needed, and the computational savings allow for longer time scales to be studied. Free-boundary simulations are used to explore the spatial dynamics; in particular, the dynamics of the thermal quench and how heat gets deposited on the material wall.

\section{TEMPORAL GROWTH}

Long wavelength instabilities in fusion plasmas have often been described within the framework of MHD theory. These instabilities are often categorized by the physics in the generalized Ohms law required for the instability to be described (e.g., ideal, resistive, neoclassical, drift). The types of instabilities have different time scales associated with their growth, with the ideal modes growing on the Alfvénic time scale, and the other modes having a time scale that is a hybrid of the Alfvénic and resistive time scales. In addition to the time scales of the mode growth, the marginal stability point as calculated by linear eigenvalue codes is frequently used to determine the type of mode in theory-experimental comparisons. $^{4}$

However, this traditional type of analysis neglects how the plasma reached an unstable equilibrium. Recently, an analytic theory ${ }^{3}$ has been put forth to describe the growth of an instability being driven through the marginal stability point. Assuming that the free energy of the mode is proportional to internal energy as measured by $\beta$,

$$
\omega^{2}=\frac{\delta W}{\delta K} \sim-\gamma_{\mathrm{MHD}}^{\prime 2}\left(\frac{\beta}{\beta_{\text {crit }}}-1\right) .
$$

Assuming a slow heating rate so that the heating may be approximated as a linear increase in $\beta$ with a heating rate $\gamma_{h}$ near the marginal point, $\beta(t)=\beta_{\text {crit }}\left(1+\gamma_{h} t\right)$, one obtains a growth rate that depends on the heating rate with the resultant mode growing faster than exponential:

$$
\xi=\xi_{0} \exp \left[(t / \tau)^{3 / 2}\right] .
$$

The time constant of the mode is a hybrid of the measure of the variation of the growth rate with beta and the heating time scale:

$$
\tau \equiv \frac{(3 / 2)^{2 / 3}}{\left(\gamma_{\mathrm{MHD}}^{\prime}\right)^{2 / 3} \gamma_{h}^{1 / 3}} .
$$

As the limit of either $\gamma_{\text {MHD }}^{\prime}$ or $\gamma_{h}$ goes to zero, the mode does not grow because it is exactly at the marginal point.

This heuristic analytic theory was successfully used to explain many of the features of DIII-D discharge 87009 which disrupted during neutral-beam heating, ${ }^{3}$ including the time scale which was deemed to be too slow for an ideal mode. An interesting part of this derivation is the use of linear theory to model what experimentalists observe to be in the nonlinear regime. To further test this theory and gain additional insight into the nonlinear behavior, discharge 87009 was modeled using the nonlinear resistive MHD equa-

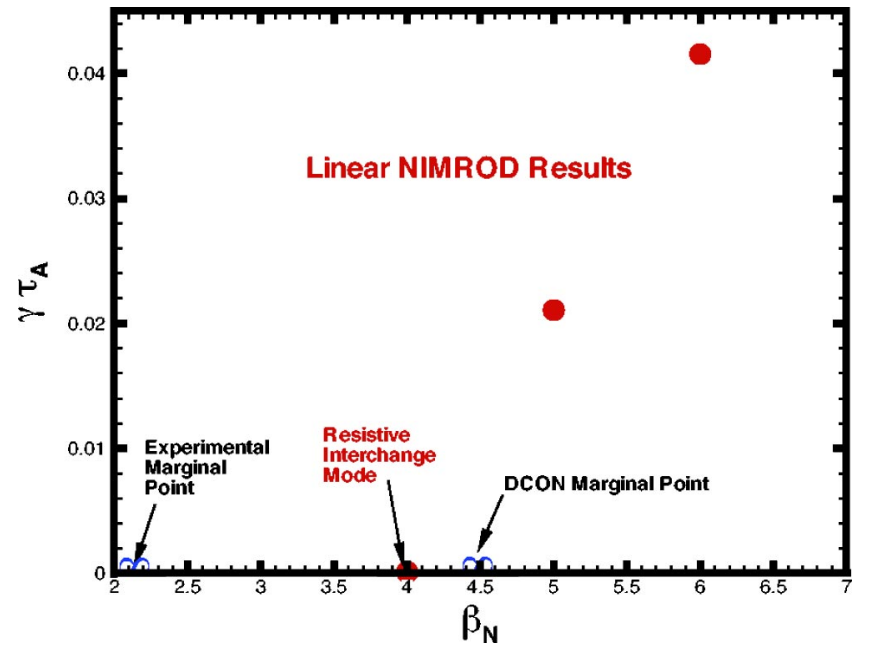

FIG. 2. (Color online). Linear NIMROD runs used in conjunction with DCON determine the marginal stability point with pressure that is larger than the experiment. Nonlinear NIMROD runs started with an equilibrium at $\beta_{N}$ $=4.70$.

tions with anisotropic heat conduction with an equilibrium with similar pressure and safety factor profiles as the actual discharge at $1681.7 \mathrm{~ms}$.

Before running a self-consistent nonlinear simulation with heating, it is necessary to begin the simulation near the ideal marginal stability point. Because having a conducting wall placed on the last closed flux is stabilizing, the plasma pressure was raised to the ideal marginal stability point by self-similarly increasing the plasma pressure profile. To determine the critical beta with sufficient accuracy, the equilibrium was varied from $\beta_{N}=4.0$ to $\beta_{N}=5.0$ in increments of $\Delta \beta_{N}=0.05$. The ideal linear stability of the equilibria was tested with DCON $^{16}$ to determine plasma stability to ideal modes using a generalized version of Newcomb's criterion. ${ }^{17}$ Because linear calculations with NIMROD take much longer than DCON to determine ideal stability, using DCON on a large number of equilibria is generally preferred. The ideal marginal stability point was found by DCON to be $\beta_{N}=4.45$. Linear NIMROD simulations found resistive interchange modes at $\beta_{N}=4.0$, and the extremely robust growth rates expected of ideal instabilities at $\beta_{N}=5.0$ and $\beta_{N}=6.0$ as shown in Fig. 2 . Because the growth of the mode at $\beta_{N}=4.45$ is very slow, we consider it to be computationally stable (with regards to ideal instabilities) and we chose $\beta_{N}=4.70$ as the starting point for our calculations.

To model the heating of the plasma, we apply a heating source that increases the equilibrium pressure self-similarly

$$
\frac{\partial p}{\partial t}=\cdots+\gamma_{h} p_{\mathrm{eq}}
$$

As the plasma heats, the flux surfaces shift outward, but the heating is still maximum at the old magnetic axis. Because our heating rate is slow compared to the growth of the mode, but still much faster than the resistive decay time $\left(\gamma_{\text {MHD }}^{\prime}\right.$ $\left.\gg \gamma_{h} \gg 1 / \tau_{R}\right)$, the assumptions of the analytic theory ${ }^{3}$ are satisfied. Note that throughout the simulations, the finite- 


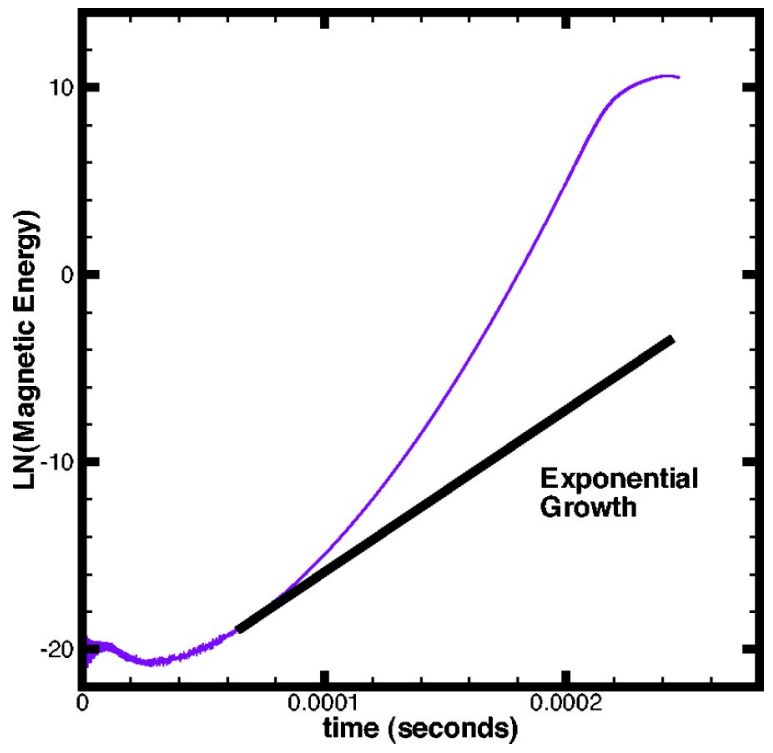

FIG. 3. (Color online). The $n=1$ perturbation grows faster than exponential as predicted by analytic theory.

element grid of NIMROD is aligned to the equilibrium magnetic field and does not move.

The NIMROD simulations were run with Lundquist number, $S=10^{6}$, Prandtl number (ratio of normalized resistivity to kinematic viscosity $\operatorname{Pr}=200$ with heating rates of $\gamma_{h}$ $=10^{-3} \mathrm{~s}^{-1}$ and $\gamma_{h}=10^{-2} \mathrm{~s}^{-1}$. A finite-element grid in the poloidal plane with 128 radial vertexes and 64 poloidal vertexes was used with bicubic finite elements. ${ }^{9}$ The toroidal direction is discretized using the pseudo-spectral method using the $n=0$ and $n=1$ modes. Because the vacuum region is not included in these simulations, the increase in beta to make the $n=1$ mode unstable results in the higher $n$ modes to be substantially destabilized. Because two-fluid effects stabilize these modes but introduce a substantial time step limitation due to the dispersive Whistler wave, only the first two modes are kept. Our results are only qualitatively correct in the fully nonlinear regime, but the goal is to compare with the quasi-linear analytic theory. The results of NIMROD simulations with $\gamma_{h}=10^{-3} \mathrm{~s}^{-1}$ are shown in Fig. 3. As predicted by the analytic theory, the growth of the mode is faster than exponential. As represented by the straight lines in the fitting shown in Fig. 4, the growths satisfy Eq. (8) well into the nonlinear regime. Using the slopes of the lines in Fig. 4 to determine the time constant for each heating rate gives a fit to the time constant of

$$
\tau \sim \gamma_{\text {MHD }}^{\prime-0.72} \gamma_{h}^{-0.28},
$$

which agrees well with the analytic prediction given by Eq. (9).

In doubly periodic geometry, any non-axisymmetric magnetic harmonic that has a component of the magnetic field perpendicular to the axisymmetric flux surfaces at the harmonics rational surface causes a change in topology. Perturbations that satisfy $\mathbf{B}_{m n} \cdot \hat{\mathbf{n}}=\mathbf{B}_{m n} \cdot \boldsymbol{\Delta} \psi_{0} /\left|\boldsymbol{\Delta} \psi_{0}\right| \neq 0$ where $n$ is the normal vector at the $q=m / n$ rational surface, break topology and are termed tearing-parity modes. Modes that have zero resonant normal components are termed

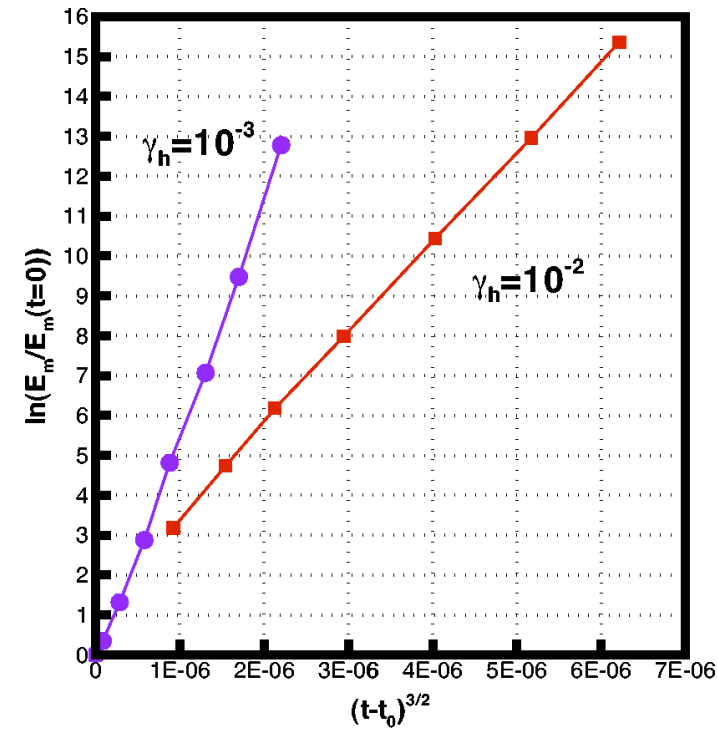

FIG. 4. (Color online). Plotting the $\log$ of the magnetic energy, $E_{m}$, vs the normalized time raised to $3 / 2$ power shows excellent agreement with the analytically predicted scaling behavior as evidenced by the straight lines.

interchange-parity modes. ${ }^{18}$ Due to the frozen flux theorem, ideal instabilities necessarily are interchange-parity modes. As ideal instabilities grow in amplitude, they distort the flux surfaces, but do not cause confinement degradation because the topology is unchanged. In Fig. 5, the normal components of the dominant harmonics are plotted along with the safety factor for time $t-t_{0}=217 \mu \mathrm{s}$. The harmonics are calculated with reference to the new flux surfaces that have shifted due to the heating of the plasma (the magnetic axis has shifted 2 $\mathrm{cm}$ at this time). The safety factor profile is the same (within numerical accuracy) as the equilibrium profile, which is consistent with no reconnection and the slow heating assumption of the flux-conserving tokamak. ${ }^{19}$ At this time, the $n=1$ perturbation is approximately two orders of magnitude smaller than the equilibrium toroidal field; thus it is in the nonlinear regime. Even in the nonlinear regime, the harmonics have similar structure as the linear eigenfunctions, only scaled to larger amplitudes, explaining the good agreement of the linear ideal MHD eigenfunctions and experimental data ${ }^{4}$ even though the experiment is in the nonlinear regime.

\section{DYNAMICS OF HEAT TRANSPORT}

To understand how a large, nonlinear ideal perturbation can lead to a thermal quench, freeboundary simulations were performed by initiating from an equilibrium based on the best equilibrium reconstruction at $1675 \mathrm{~ms}$. Because starting below the critical ideal MHD threshold adds computational cost to an already expensive calculation, equilibrium pressure was raised self-consistently by $8.7 \%$ above the best equilibrium reconstruction to place the plasma beta above the ideal MHD threshold. The plasma equilibrium and a cross-sectional plot of the plasma is shown in Fig. 1.

The simulations presented were run with a temperaturedependent resistivity normalized such that the Lundquist number in the core plasma was $S=10^{5}$. A ratio of $\kappa_{\|} / \kappa_{\perp}$ $=10^{8}$ was held constant throughout the computational do- 

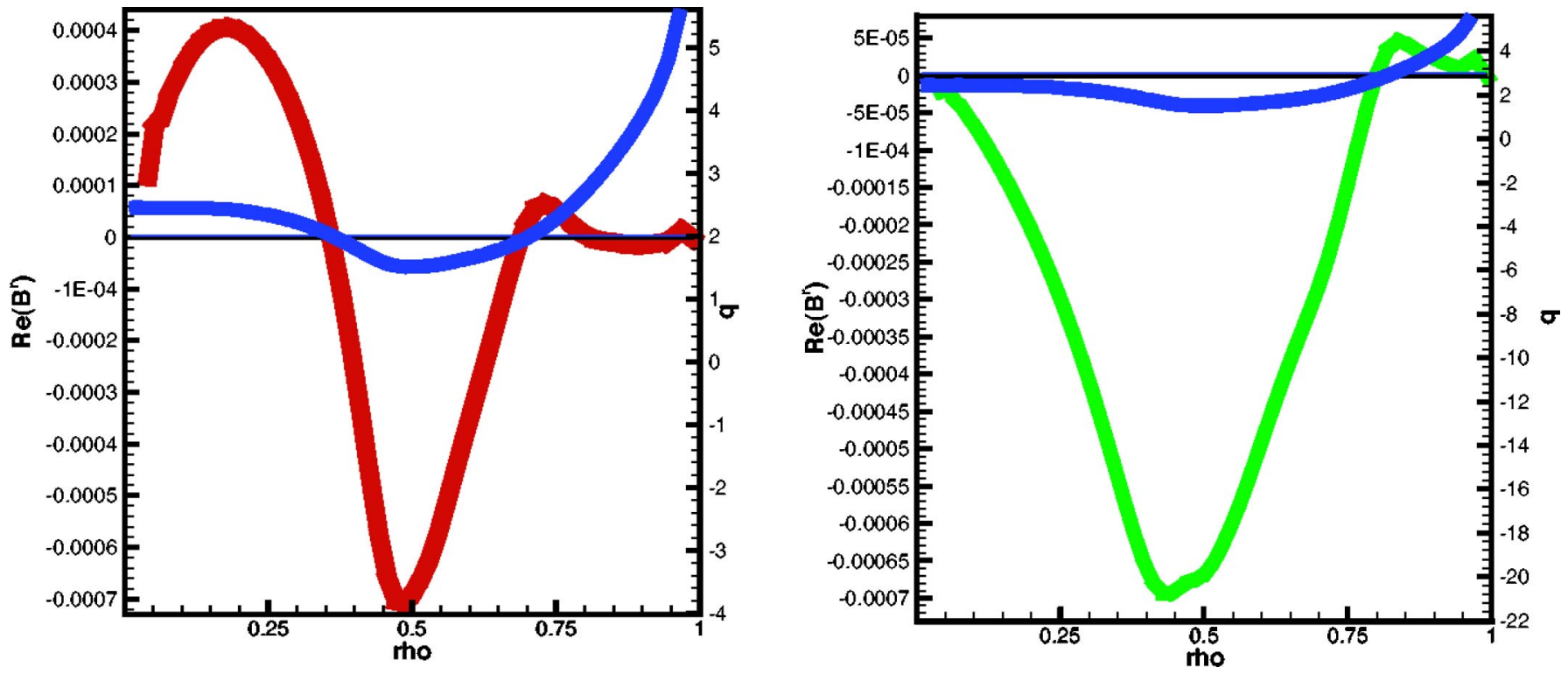

FIG. 5. (Color online). The 2/1 (right) and 3/1 (left) harmonics of $B_{n}$ have zero components at their resonant surfaces indicating no change of topology.

main. The use of constant coefficients results in the parallel conductivity being underestimated in the core region and overestimated in the halo region, but greatly simplifies analysis of the complex dynamics. The boundary conditions are applied at the vacuum vessel (modeled as a perfectly conducting wall), and not the first material wall, as seen in Fig. 1. The normal component of the magnetic field is held constant at the conducting surface. For the density, velocity, and temperature, the boundary conditions are also applied at the vacuum vessel instead of the more physical limiter surface. This is done for two reasons: (1) applying the natural boundary conditions at the limiter would give no density flux across the limiter, which is less physical than our current method of applying the natural boundary conditions at the vacuum vessel and allowing a mass flux across the limiter; (2) on the time scales of the mode growth and nonlinear evolution, impurities would not have time to penetrate into the plasma. Thus, complicated plasma-wall interactions are unimportant for this simulation.

In Fig. 6, the global parameters of internal energy and plasma current from the NIMROD simulation are shown. The plasma energy decreases by two-thirds in approximately 200 $\mu \mathrm{s}$, in qualitative agreement with the experiment. As the internal inductance changes due to the reconnection processes, the plasma current increases as is also observed in the experiment. To explain the processes leading to the loss of energy confinement, a series of visualizations is presented. In Fig. 7, the initial starting point of the NIMROD simulation is shown. The temperature isosurfaces show a reversed temperature profile due to the peaked pressure profile and broader density profile. The magnetic field line plotted is colored according to the temperature with the brightness of the nodes indicating the distance along the field line. Finally, the DIII-D limiter wall, corresponding to the axisymmetric version of the limiter shown in (1), is shown with the heat flux contours plotted on this surface. At this early point in time, the heat flux is small and cannot be seen.
As the temperature evolves, the first notable macroscopic feature in the temperature isosurfaces is the appearance of a $2 / 1$ island (Fig. 8), which occurs at $505 \mu$ s from the beginning of the simulation. As seen in the Poincare surface of section in Fig. 8, at this time step there are two 2/1 rational islands, a large $3 / 1$ island, and the edge is stochastic. As can be seen from the safety factor profile in Fig. 1, two 2/1 rational surfaces exist with the outer surface having lower shear. The temperature only shows the inner $2 / 1$ island be-
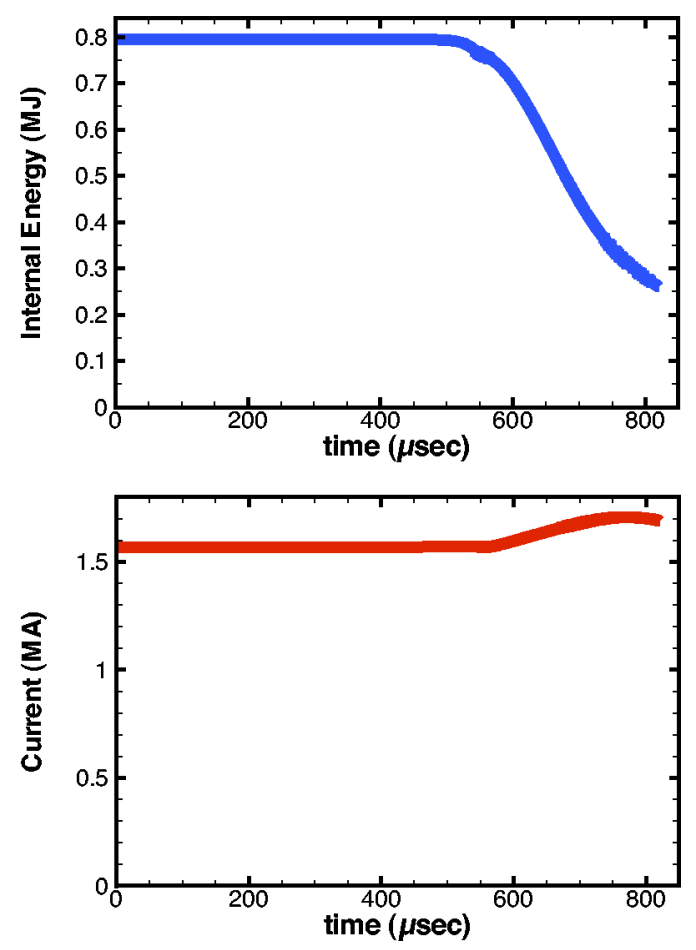

FIG. 6. (Color online). The plasma loses two-thirds of its internal energy in $200 \mu \mathrm{s}$ in qualitative agreement with experiment. As the plasma expands, the internal inductance changes causing the current to increase. 


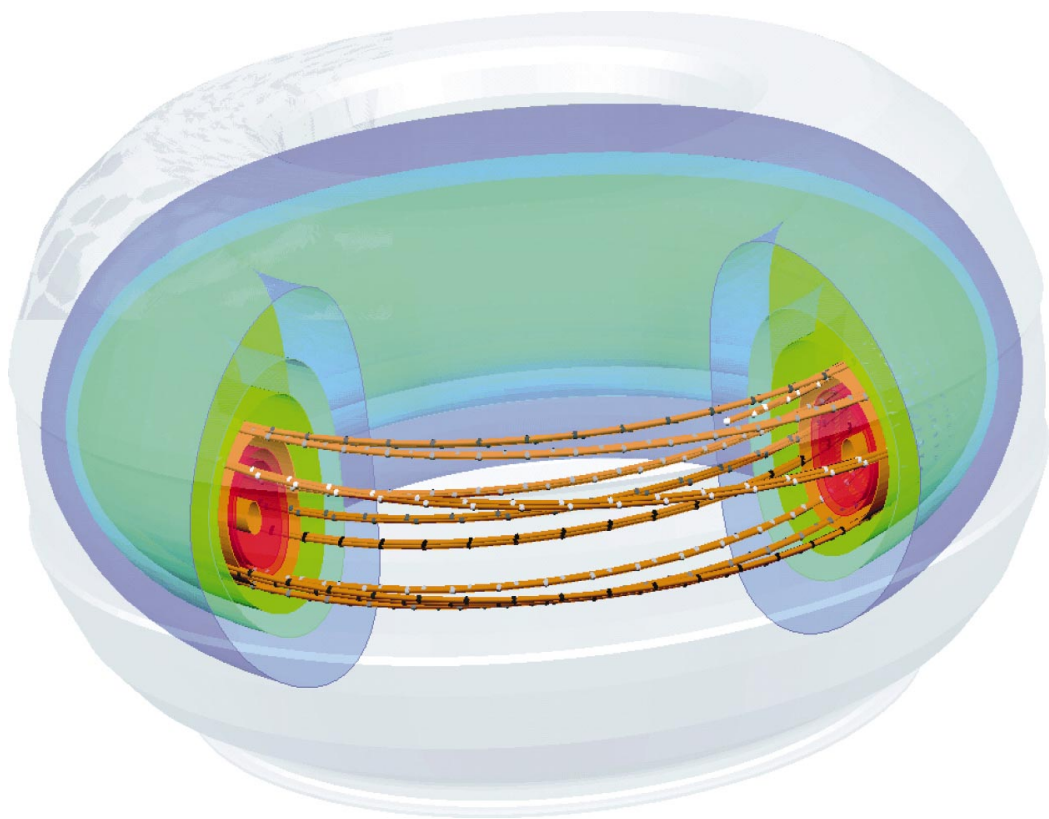

FIG. 7. (Color). The axisymmetric starting point for the three-dimensional simulations shows the temperature isosurfaces, two magnetic field lines, and the heat flux on the divertor, which at this point in time is negligible.

cause of the competition of the parallel and perpendicular heat conduction near rational surfaces. ${ }^{20}$ The scale length of this equilibration competition increases with decreasing shear. We note that more conventional DIII-D shear profiles have an equilibration length scale of $4 \mathrm{~cm}$ for $\kappa_{\|} / \kappa_{\perp}=10^{8}$. The fact that the edge went stochastic first is a generic feature of the magnetic nulls at the $X$ point. Because the pressure profile is a peaked $L$-mode profile (Fig. 1), little stored energy is lost in this phase of the disruption.

As the islands grow and overlap, the core becomes stochastic as well. At $546 \mu$ s into the simulation, the core region is largely stochastic as shown in Fig. 9. The field line, which was started at the same location as in Fig. 8, is more volume filling; however, it is confined and does not strike the wall. The heat flux on the limiter is increasing as evidenced by the contour plot on the limiter wall. At this time, $96 \%$ of the internal energy is still contained by the plasma.
At time of maximum normal heat flux on the material wall $\left(t-t_{0}=639 \mu \mathrm{s}\right)$, the heat flux is localized toroidally and poloidally as seen in Fig. 10. At this point in time, $87 \%$ of the internal energy is still confined. Because the time of maximum heat flux on the wall occurs well after the core has become stochastic, and because it is of greatest interest for an engineering point of view, a more complete investigation of the heat transport at this time is presented.

In Fig. 11, the isosurfaces corresponding to $47 \%$ of the peak value of the magnitude of the perpendicular heat flux is shown (because $\left|\mathbf{q}_{\perp}\right|^{2}=-\kappa_{\perp} \mathbf{q}_{\perp} \cdot \nabla T$ this can also be viewed as a measure of the perpendicular heat flux flowing through a temperature isosurface). As seen in the figure, a large amount of heat transport is on the inboard side and in the core. In addition, two small isosurfaces are located farther out on the outboard side as shown in the lower part of the figure. This particular value of heat flux magnitude was chosen for visu-

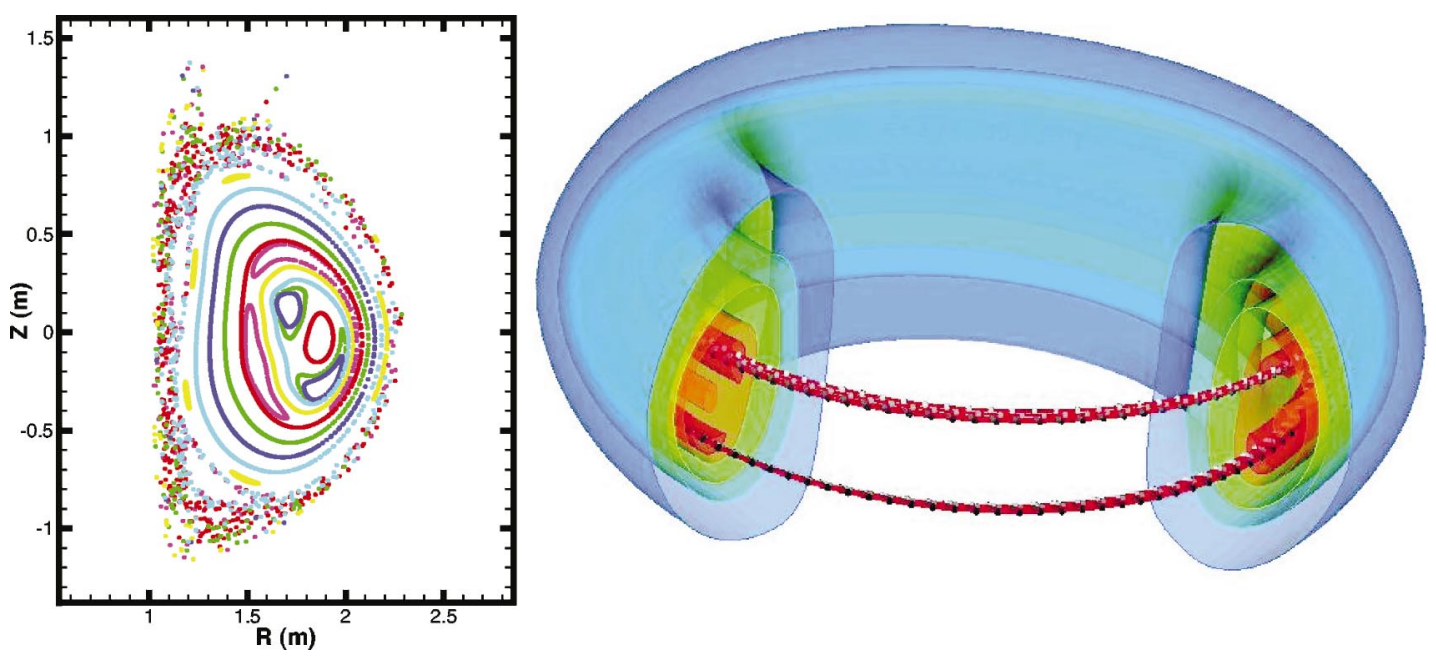

FIG. 8. (Color). At $t-t_{0}=464 \mu$ s, multiple islands can be observed in the Poincare surface of section (left) and the edge region is largely stochastic. The temperature isosurfaces (right) only show the inner $2 / 1$ magnetic island. 

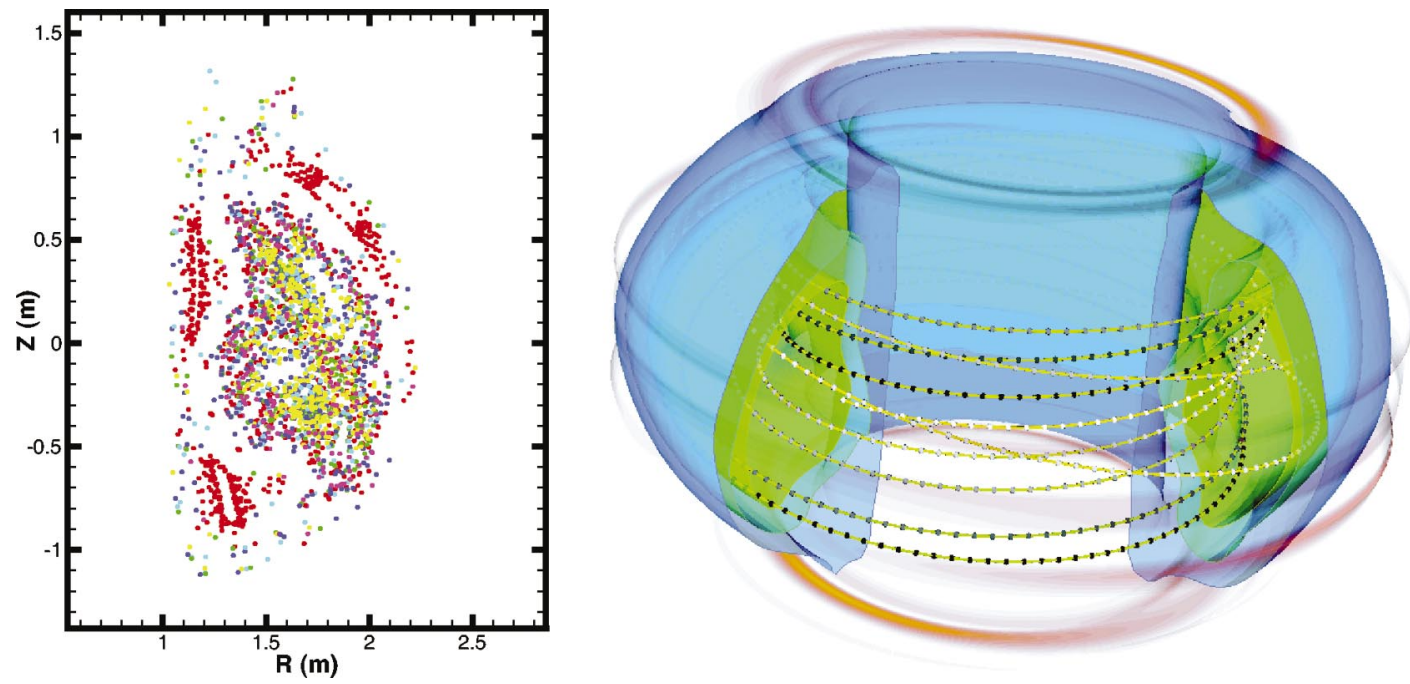

FIG. 9. (Color). At $t-t_{0}=546 \mu \mathrm{s}$, the core region is largely stochastic although the field lines are confined. The heat flux is rising on the limiter although it is not at its maximum value.

alization because it is the first time an isosurface appears this far out on the outboard mid-plane. All larger magnitudes of heat flux occur in the core region. Four field lines are initialized near this location as shown in the inset of Fig. 11. In Fig. 12, the integrated field lines are shown with the color denoting the total length of the field lines. The red field line, which was initialized from a point inside of the isosurfaces, is confined completely within the plasma, and the blue field lines strike both the top and bottom divertors. Because the heat transport along magnetic field lines is much more rapid than heat transport across field lines, any heat flux that reaches the open field lines is able to quickly equilibrate and reach the plasma boundary. The localization of the normal heat flux on the divertor is a consequence of the localization of the perpendicular heat flux within the core plasma. As can be seen by the isosurfaces of heat flux, the most important localization is that which occurs near the transition between the open and closed field lines. The heat flux that is localized in the core plasma remains relatively well confined even though the field is stochastic. An examination of the red field line shows that it is related to the heat flux isosurfaces in the interior, and possibly contributes to the appearance of the outer hot spot.

The contour plots of the normal heat flux on the top and bottom divertors in Fig. 12 also show the movement of the original divertor strike points. The brightest spots indicate the location of the original strike points. Moving $180^{\circ}$ away from this spot shows a bifurcation in the heat flux impinging upon the wall. The distance between these two peaks at this toroidal location is $5 \mathrm{~cm}$. A comparison of Fig. 9 and Fig. 10 indicates that this bifurcated structure appeared early in the plasma discharge. The $n=1$ dominance of this bifurcation is due to the initial $n=1$ perturbation driving the edge boundary distortion as the edge goes stochastic. The implication is that higher $n$ perturbations, such as edge localized modes (ELMs), would presumably give even more toroidal, and
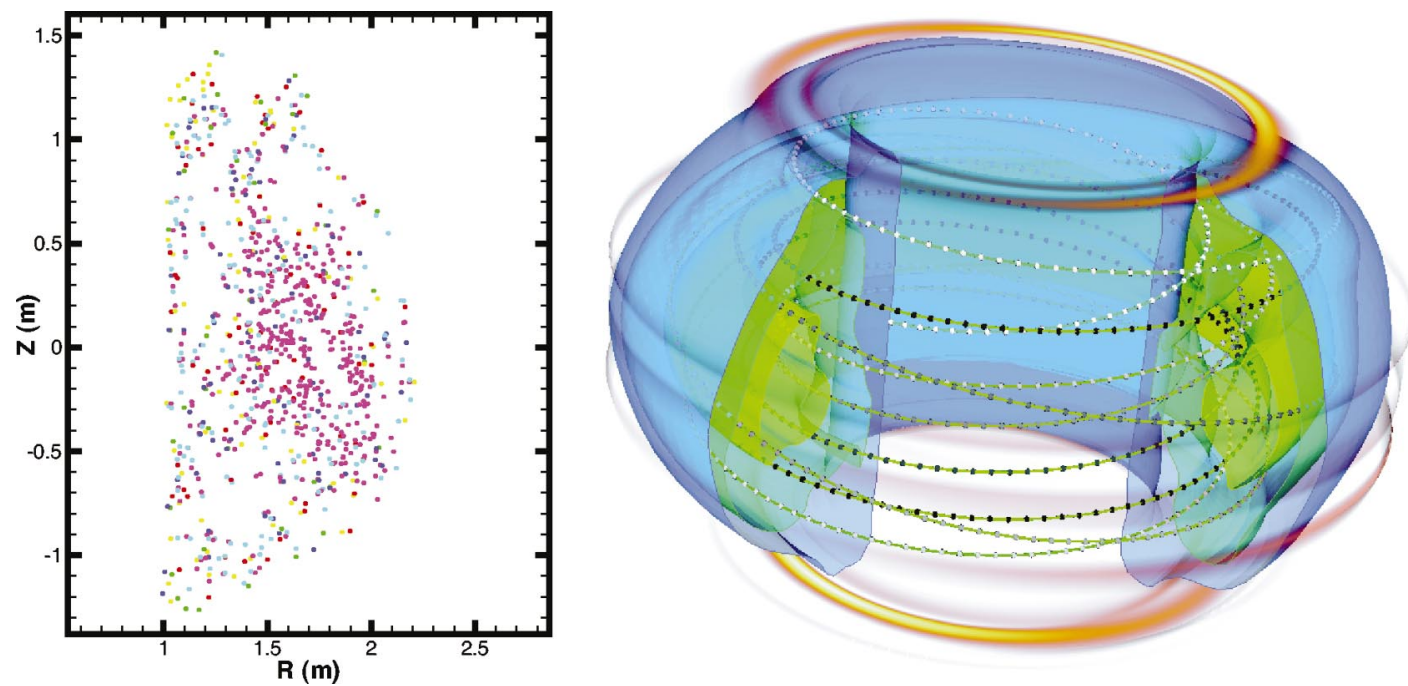

FIG. 10. (Color). The heat flux being deposited on the upper and lower divertors is localized both toroidally and poloidally. At this time, the heat flux loading on the wall is maximum, and the field is completely stochastic in the core. 

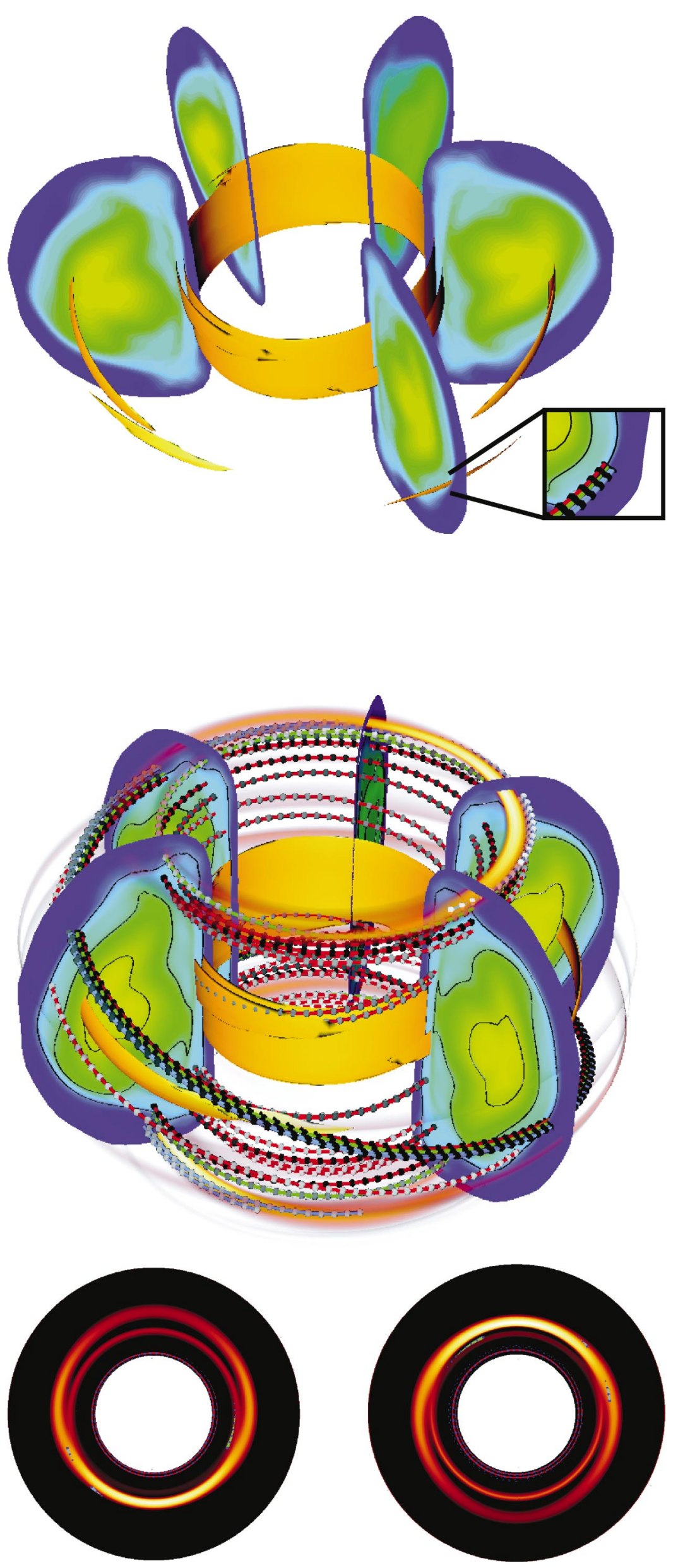

FIG. 11. (Color). Isosurfaces of the magnitude of the perpendicular heat flux is plotted to show where the perpendicular heat flux is flowing across the temperature isosurfaces. The field lines shown in Fig. 12 are started near the outer isosurfaces as shown by the inset.
FIG. 12. (Color). Field lines started near the openclosed boundary show different contributions to the peak heat flux loading on the wall based on position near the heat flux isosurface. The field lines are colored by their total length with the red field line being completely confined and the blue field lines striking the top (shown in the lower left) and bottom (shown in the bottom right) divertors. 
hence poloidal, variation. Because tokamaks are designed to handle their maximum heat loads based on the equilibrium strike points, understanding the movement of the peak wall loading away from their wall is an important part of these studies.

\section{DISCUSSION AND CONCLUSIONS}

Despite the heuristic nature of the analytic derivation of mode growth being driven through an ideal marginal instability point, the NIMROD simulations show that the analytic scaling given by Eq. (8) gives an excellent description of the mode growth even into the nonlinear regime. For ideal modes, where the mode amplitudes can grow quite large without a change in magnetic topology, the fundamental assumptions of the analytic theory hold into the nonlinear regime. The simulations emphasize the point of the importance of simulating not only the instability, but also the mechanism by which the plasma has evolved to that state. Unfortunately, simulations near the marginality point are the most difficult to simulate because of the slowness of the mode growth. How resistive instabilities, which are unstable below the ideal marginal stability point, influence the evolution of the discharge to the unstable state is an open question. Investigating this question, will be studied in future work.

The free boundary simulations that explored the realistic DIII-D geometry and heat flux deposition agrees qualitatively with many features of the thermal quench phase of plasma disruptions. The macroscopic loss of energy confinement time of $200 \mu$ s is in qualitative agreement with experiment, as is the concomitant rise in the plasma current due to the change in internal inductance. The edge went stochastic first, as would be expected given the $X$ points and the closeness of the rational surfaces in that region. Because of the peaked, $L$-mode pressure profile, little of the stored energy was lost in the early phase of the disruption. Future work will include the extension of these simulation diagnostic techniques for studying heat transport to ELM simulations where significant stored energy is located near the separatrix.

The localization of the heat flux, both toroidally and poloidally, has been observed in divertor temperature measurements in similar experiments. The heat flux localization arises because the original mode distorts the plasma surfaces and localizes the perpendicular heat flux. As the heat flux increases near the boundary between open and closed field lines, the parallel heat conduction rapidly carries it to the wall. Although the use of a diffusive heat transport model with constant coefficients is a relatively crude model for equilibration of temperature along field lines and probably exaggerates the importance of the open-closed boundary, avoiding the complications of temperature-dependent coefficients is useful for gaining a qualitative understanding of the overall dynamics of heat transport in a complicated magnetic topology. Future work will include more accurate modeling of the heat flux, including temperature-dependent Braginskii coefficients, use of Landau-fluid closures, ${ }^{21,22}$ and a kinetic calculation of heat flux. ${ }^{23,24}$

Much of the success of the modeling this discharge is due to the peaked, $L$-mode pressure profile of the equilibrium studied. Because the pressure gradient was peaked inside the plasma, the resultant mode was largely internal and resulted in little movement of the plasma boundary. Complicated plasma-wall interactions were not required for the obtained agreement. Also, because the time scale of the ideal mode growth is rapid compared to the time scale of impurity penetration, the need for impurity modeling is unnecessary. For disruptions caused by resistive wall modes ${ }^{25}$ where mode growth is both slow and primarily external, for disruption mitigation experiments where impurities are injected into the plasma, ${ }^{26}$ or for density limit disruptions, ${ }^{2}$ modeling of impurities in the NIMROD code would be necessary. Current work is under way to include these effects.

Future simulations will try to drive the free-boundary simulations through the stability point in the same way the fixed-boundary equilibria were driven. By more accurately modeling the time-dependence, the heat flux closure, and more realistic diffusivity parameters, direct quantitative comparisons of simulation results to experimental diagnostics can be made. By improving our understanding of disruptions, intuition can be gained to guide the experiments, and allow the use of numerical experiments to explore how factors such as external magnetic configuration can be used to mitigate the damage caused by major disruptions.

\section{ACKNOWLEDGMENTS}

This work was strongly motivated by the work of and discussions with Dr. J.D. Callen. Dr. M.-S. Chu, Dr. A. Turnbull, and Dr. D. Brennan provided several useful discussions and the equilibria used in this study. Dr. D. Whyte and Dr. A. Tarditi offered many useful suggestions. The visualization done in this work was done in collaboration with Dr. A. Sanderson utilizing the SCIRun Problem Solving Environment $^{27}$ under the Fusion Collaboratory SciDAC. SCIRun was made possible by the NIH/NCRR Center for the Bioelectric Field Modeling, Simulation, and Visualization, P41-RR12553.

The Office of Fusion Energy Sciences sponsored this work under Contract No. DE-FC03-02ER54666.

\footnotetext{
${ }^{1}$ R. Aymar, P. Barabaschi, and Y. Shimomura, Plasma Phys. Controlled Fusion 44, 519 (2002).

${ }^{2}$ P. Taylor, A. Kellman, T. Evans, D. Gray, D. Humphreys, A. Hyatt, T. Jernigan, R. Lee, J. Leuer, S. Luckhardt et al., Phys. Plasmas 6, 1872 (1999).

${ }^{3}$ J. Callen, C. Hegna, B. Rice, E. Strait, and A. Turnbull, Phys. Plasmas 6, 2963 (1999).

${ }^{4}$ A. Turnbull, D. Brennan, M. S. Chu et al., in Proceedings of the 18th International Conference on Fusion Energy (Sorrento, Italy, 2000), (IAEA, 2001).

${ }^{5}$ E. Strait, Phys. Plasmas 1, 1415 (1994).

${ }^{6}$ M. Chu, J. Greene, L. Lao, R. Miller, A. Bondeson, O. Sauter, B. Rice, E. Strait, T. Taylor, and A. Turnbull, Phys. Rev. Lett. 77, 575 (1996).

${ }^{7}$ A. Popov, V. S. Chan, M. S. Chu, Y. Q. Liu, B. W. Rice, A. D. Turnbull, Phys. Plasmas 8, 3605 (2001).

${ }^{8}$ J.-N. Leboeuf, V. Lynch, and B. Carreras, Phys. Plasmas 8, 3358 (2001). ${ }^{9}$ C. Sovinec, A. Glasser, T. Gianakon, D. Barnes, R. Nevel, S. Kruger, S. Plimpton, A. Tarditi, M. Chu, and the NIMROD Team, J. Comput. Phys. 195, 355 (2004)

${ }^{10}$ J. Birn, J. Drake, M. Shay, B. Rogers, R. Denton, M. Hesse, M. Kuznetsova, Z. Ma, A. Battacharjee, A. Otto et al., J. Geophys. Res. 106, 3715 (2001).

${ }^{11}$ J. DeLucia, S. Jardin, and A. Todd, J. Comput. Phys. 37, 183 (1980).
} 
${ }^{12}$ W. Park, E. Fredrickson, A. Janos, J. Manickam, and W. Tang, Phys. Rev. Lett. 75, 1763 (1995).

${ }^{13}$ K. Shin, Y. Lim, C. Shon, and J. Lee, IEEE Trans. Plasma Sci. 27, 110 (1999).

${ }^{14}$ H. Strauss, W. Park, E. Belova, G. Fu, and L. Sugiyama, Nucl. Fusion 39, 2069 (1999).

${ }^{15}$ A. Aydemir, Phys. Plasmas 8, 3411 (2001).

${ }^{16} \mathrm{~A}$. Glasser, (private communication).

${ }^{17}$ W. Newcomb, Ann. Phys. 10, 362 (1960).

${ }^{18}$ B. Coppi, J. Greene, and J. Johnson, Nucl. Fusion 6, 101 (1966).

${ }^{19}$ R. Dory and Y.-K. Peng, Nucl. Fusion 17, 21 (1977).

${ }^{20}$ R. Fitzpatrick, Phys. Plasmas 2, 825 (1995).

${ }^{21}$ G. Hammett and F. Perkins, Phys. Rev. Lett. 64, 3019 (1990).
${ }^{22}$ P. Synder, G. Hammett, and W. Dorland, Phys. Plasmas 4, 3974 (1997).

${ }^{23}$ E. Held, J. D. Callen, C. C. Hegna, and C. R. Sovinec, Phys. Plasmas 8, 1171 (2001).

${ }^{24}$ E. Held, J. D. Callen, C. C. Hegna, C. R. Sovinec, T. Gianakon, and S. Kruger, Phys. Plasmas 11, 2419 (2004).

${ }^{25}$ A. Garafalo, T. Jensen, L. Johnson, R. LaHaye, G. A. Navratil, M. Okabayashi, J. Scoville, E. Strait, D. Baker, J. Bialek et al., Phys. Plasmas 9, 1997 (2002).

${ }^{26}$ E. Hollmann, D. Gray, D. Whyte, A. Pigarov, S. Krasheninnikov, J. Boedo, and D. Humphreys, Phys. Plasmas 10, 2863 (2003).

${ }^{27}$ SCIRun, SCIRun: A scientific computing problem solving environment (2002), scientific Computing and Imaging Institute (SCI), URL http:// software.sci.utah.edu/scirun.html. 